\title{
Fluorescence Lifetime Imaging Combined with Conventional Intravascular Ultrasound for Enhanced Assessment of Atherosclerotic Plaques: an Ex Vivo Study in Human Coronary Arteries
}

\author{
Hussain Fatakdawala $^{1}$ • Dimitris Gorpas ${ }^{1} \cdot$ John W. Bishop ${ }^{2} \cdot$ Julien Bec $^{1}$ • \\ Dinglong $\mathrm{Ma}^{1}$ • Jeffrey A. Southard ${ }^{3} \cdot$ Kenneth B. Margulies ${ }^{4} \cdot$ Laura Marcu $^{1}$ \\ Received: 17 February 2015 / Accepted: 15 April 2015 /Published online: 1 May 2015 \\ (C) The Author(s) 2015. This article is published with open access at Springerlink.com
}

improved evaluation of plaques that may subsequently aid in guiding coronary intervention.

Keywords Atherosclerosis · Cardiovascular diseases · Intravascular imaging $\cdot$ Fluorescence lifetime imaging · Intravascular ultrasound

\begin{tabular}{|c|c|}
\hline \multicolumn{2}{|c|}{ Abbreviations } \\
\hline FLIm & Fluorescence lifetime imaging \\
\hline IVUS & Intravascular ultrasound \\
\hline PCI & Percutaneous coronary intervention \\
\hline OCT & Optical coherence tomography \\
\hline NIRS & Near-infrared reflectance spectroscopy \\
\hline TCFA & Thin-cap fibroatheroma \\
\hline ThCFA & Thick-cap fibroatheroma \\
\hline LAD & Left anterior descending \\
\hline UV & Ultraviolet \\
\hline $\mathrm{KHz}$ & Kilohertz \\
\hline $\mathrm{MHz}$ & Megahertz \\
\hline ns & Nanosecond \\
\hline rpm & Rotations per minute \\
\hline $\mathrm{nJ}$ & Nanojoules \\
\hline ROI & Region of interest \\
\hline $\mathrm{CD}$ & Clusters of differentiation \\
\hline UI & Ultrasound intensity \\
\hline IB & Integrated backscatter \\
\hline $\mathrm{E}$ & Energy norm \\
\hline IT & Intimal thickness \\
\hline $\mathrm{R}$ & Radial distance \\
\hline $\mathrm{CH} 1$ & Channel 1 \\
\hline $\mathrm{CH} 2$ & Channel 2 \\
\hline $\mathrm{CH} 3$ & Channel 3 \\
\hline LFA & Lipid-rich core of fibroatheroma \\
\hline SVM & Support vector machine \\
\hline
\end{tabular}




$\begin{array}{ll}\text { DIT } & \text { Diffuse intimal thickening } \\ \text { PIT } & \text { Pathological intimal thickening } \\ \text { FC } & \text { Fibrocalcific plaque } \\ \text { FT } & \text { Fibrotic tissue } \\ \text { ThCFAM } & \begin{array}{l}\text { Thick-cap fibroatheroma with macrophage/ } \\ \text { lymphocyte }\end{array} \\ \text { ANOVA } & \text { Analysis of variance }\end{array}$

\section{Introduction}

Majority of sudden acute cardiovascular events are caused due to rupture or erosion of vulnerable plaques [1]. The identification of these plaques can aid in the advancement of interventional techniques and pharmacological therapies to reduce such events. Since angiography provides limited information about the nature of the plaque occluding a vessel, percutaneous coronary interventions (PCIs) assisted with imaging techniques have been realized as an important tool for studying plaque progression and pathology in animals in vivo and in patients.

Optical coherence tomography (OCT) and intravascular ultrasound (IVUS) are the two most common intravascular imaging techniques used in the catheterization laboratory [2] with near-infrared spectroscopy (NIRS) recently introduced into clinical practice as well [3]. IVUS is not able to detect rupture-prone thin-cap fibroatheromas (TCFAs) due to limited spatial resolution $(>100 \mu \mathrm{m})$. Conversely, OCT has a spatial resolution of 10-20 $\mu \mathrm{m}$ and can detect thin caps [4] as well as assess inflammation [5] but has limited imaging penetration depth and cannot reliably determine the size of deep plaque cores or outward remodeling, an important indicator of plaque vulnerability. NIRS has the ability to detect lipid cores but has limited sensitivity for other proteins of interest such as collagen and elastin. A comparison of these techniques is illustrated in Table T1 in the supplemental material. Since each modality by itself is limited in one way or another, multimodal imaging where two or more modalities complement one another to allow simultaneous assessment of plaque structure and composition would be extremely beneficial.

The overall objective of this study is to demonstrate the following: (1) imaging results from diseased human coronary vessels using a novel label-free bimodal intravascular scanning technique that combines fluorescence lifetime imaging (FLIm) and IVUS and (2) the ability of FLIm to complement conventional IVUS for improved human coronary plaque assessment via quantification of plaque composition. Previous work has shown the potential of endogenous fluorescence lifetime measurements for atherosclerotic plaque characterization in open arteries [6,7]. This paper reports the first results from human coronary arteries interrogated with such a bimodal rotational catheter that serves as an important feasibility step prior to studying plaques in an in vivo animal model as well as future human studies. We explore the ability of FLIm in detecting biochemical features (e.g., infiltration of macrophages in fibrous caps) and distinguishing between rupture-prone thin-cap fibroatheromas (TCFAs) from relatively stable thick-cap fibroatheromas (ThCFAs) that can complement morphological features derived from conventional IVUS.

\section{Methods}

\section{Specimens}

The human left anterior descending (LAD) coronary artery samples used in these studies were obtained from 16 different patients from the University of Pennsylvania with approval from the institutional review board (additional information in the supplemental material). Samples were chilled in isopentane and rapidly frozen in liquid nitrogen and shipped overnight and stored at $-80^{\circ} \mathrm{C}$.

\section{FLIm-IVUS Imaging Studies}

The FLIm-IVUS bimodal imaging system and rotational catheter (5-Fr shaft and 3-Fr imaging section) used in this study have been previously described in detail [8] and can perform sequential IVUS and FLIm helical scans. Details of the FLIm subsystem are described in the supplemental material. Briefly, it is composed of a $300-\mu \mathrm{m}$ core ultraviolet (UV)-grade silica/ silica side-viewing optical fiber (Polymicro, Phoenix, AZ) rotating at $1200 \mathrm{rpm}$ delivering UV light (Fianium, $355 \mathrm{~nm}, 80$ ps pulse width) with a repetition rate of $10 \mathrm{KHz}$ and an energy of $\sim 100 \mathrm{~nJ} / \mathrm{pulse}$. The IVUS subsystem (Atlantis SR Pro with iLab, Boston Scientific, MA, USA) is composed of a single element transducer $(40 \mathrm{MHz})$ driven at a rotation speed of $1800 \mathrm{rpm}$ with data sampled at $200 \mathrm{MHz}$. Both FLIm and IVUS imaging were performed with a pullback speed of $2 \mathrm{~mm} / \mathrm{s}$ sequentially. FLIm had an axial/angular resolution of $160 \mu \mathrm{m} / 14^{\circ}$ while IVUS had an axial/lateral resolution of $100 \mu \mathrm{m} / 200 \mu \mathrm{m}$. A 20 -mm pullback took $10 \mathrm{~s}$ for each modality. Data acquisition, saving, and processing took $6 \mathrm{~min}$ in total [8]. FLIm-IVUS co-registration was performed offline.

The specimen was imaged in a custom-built holder placed in a phosphate-buffered saline bath maintained at $37^{\circ} \mathrm{C}$. The specimen was fastened on to luers that presented a notch painted with fluorescent ink so that they were visible on both FLIm and IVUS images to allow axial and angular coregistration of both modalities (Fig. S1 in the supplemental material). Additionally, a guide wire (0.014") was also placed in the lumen that was visible on both modalities to aid in angular registration. The guide wire was immobilized by fastening it in place using the screw end of the luer receiver (Fig. S1c in the supplemental material). It must be noted that FLIm-IVUS imaging was performed in saline and not blood. 
In vivo FLIm imaging will require clearance of blood as is done during OCT imaging. Tissue processing for acquiring co-registered histology is detailed in the supplemental material. A total of 87 histological sections were cut at 2-mm intervals from all specimens. For each cut, FLIm-IVUS data were averaged from the corresponding registered frame and the two adjacent frames. All histology slides were digitized at $\times 20$ magnification (Aperio ScanScope, Aperio Technologies) for morphometric analysis.

\section{Pathological Features and Morphometric Analysis}

Eight different pathological features were characterized in this study (Table 1) following definitions published by Virmani et al. [9]. Representative histology images for various features are shown in Fig. S2 in the supplemental material. On the digitized histology images, these features were identified by a pathologist as regions of interest (ROIs). Characteristic descriptors such as the amount of collagen, elastin, lipids/cholesterol, calcium, smooth muscle cells, and macrophages/ lymphocytes were observed on pathology that allowed assigning a given ROI to one of the eight features. Significant macrophage/lymphocyte infiltration in tissue was determined by identifying the presence of $>10 \%$ clusters of differentiation (CD) 68+/CD45+ staining area in a given ROI. ROI selection and measurement of fibrous cap thickness were performed using a commercial software (Aperio ImageScope, Aperio Technologies). Corresponding co-registered imaging data were extracted by defining the same ROI on IVUS images. FLIm data were mapped onto the IVUS lumen [10] and attributed to the corresponding ROI. The ROIs on IVUS were scaled up in size proportional to the change in the scale bars between IVUS and histology when both images were co-registered. This change in ROI size between IVUS and histology is due to shrinkage in tissue after histological processing.

\section{Imaging Parameters}

A total of five IVUS-derived parameters and 42 FLIm-derived parameters were studied. The IVUS parameters included logcompressed ultrasound intensity (UI), integrated backscatter (IB) values, energy norm $(E)$, intimal thickness (IT), and radial distance $(R)$ of the ROI from the center of the IVUS transducer. The computation of these parameters have been previously reported [11] and are described in detail in the supplemental material. UI, IB, and $E$ represent the amount of sound backscattered from tissue and may allow differentiating between different tissue types.

FLIm data includes fluorescence measurements from three sub-bands with central wavelength/bandwidth of 390/40 nm (channel 1 (CH1)), 452/45 nm (channel 2 (CH2)), and 542/ $50 \mathrm{~nm}$ (channel $3(\mathrm{CH} 3)$ ), respectively. $\mathrm{CH} 1$ encompasses fluorescence predominantly from collagen, $\mathrm{CH} 2$ from elastin and NADH, and collagen and $\mathrm{CH} 3$ from lipids and lipoproteins. The FLIm parameters included intensity ratios from channels $(\mathrm{CH} 1 / \mathrm{CH} 2, \mathrm{CH} 2 / \mathrm{CH} 3$, and $\mathrm{CH} 1 / \mathrm{CH} 3)$, average lifetime values, and the 12 Laguerre coefficient values from each of the channels $\mathrm{CH} 1, \mathrm{CH} 2$, and $\mathrm{CH} 3$. The analysis of raw FLIm data for the computation of the aforementioned FLIm parameters has been described in detail previously [12] and is briefly summarized in the supplemental material. The penetration of UV light in tissue is limited to 200-250 $\mu \mathrm{m}[13,14]$. As a result, FLIm parameters are not defined for deep lipidrich core of a fibroatheroma (LFA).

\section{Statistical Analysis}

FLIm-IVUS data from ROIs were fitted to a linear mixed effects model [15] to determine statistically significant correlations between various imaging parameters and pathological features. Statistically significant imaging parameters $(p<0.05)$ were used to train a support vector machine (SVM) classifier between different pathological features. A leave-one-out cross-validation test was performed to determine the sensitivity, specificity, and positive predictive value of FLIm, IVUS, and FLIm-IVUS parameters of detecting the eight pathological conditions over the entire data set. Details of the statistical analysis are summarized in the supplemental material.

\section{Results}

Table 1 summarizes all the pathological features identified in this study along with the number of ROIs attributed to each feature from the 16 human LAD coronary specimens.

\section{FLIm-IVUS Data Visualization}

An example of three-dimensional (3-D) visualization of coregistered FLIm (CH1 data) and IVUS data from a coronary specimen highlighting different morphological and biochemical features is shown in Fig. 1 (Video1.avi in the supplemental material). Such representation of the arterial vessel can aid the physician in the detection of different plaque types by allowing simultaneous visualization of such complimentary features in a clinical setting. Changes in fluorescence lifetime values have been attributed to changes in collagen, lipid, and macrophage content $[6,7]$ and are confirmed in images shown in Fig. 2 and data summarized in Figs. 3 and 4. This information, coupled with morphological features from IVUS such as IT, hyperechoic calcified tissue, and hypoechoic lipid cores, allows identification of relevant pathology described in Table 1. 


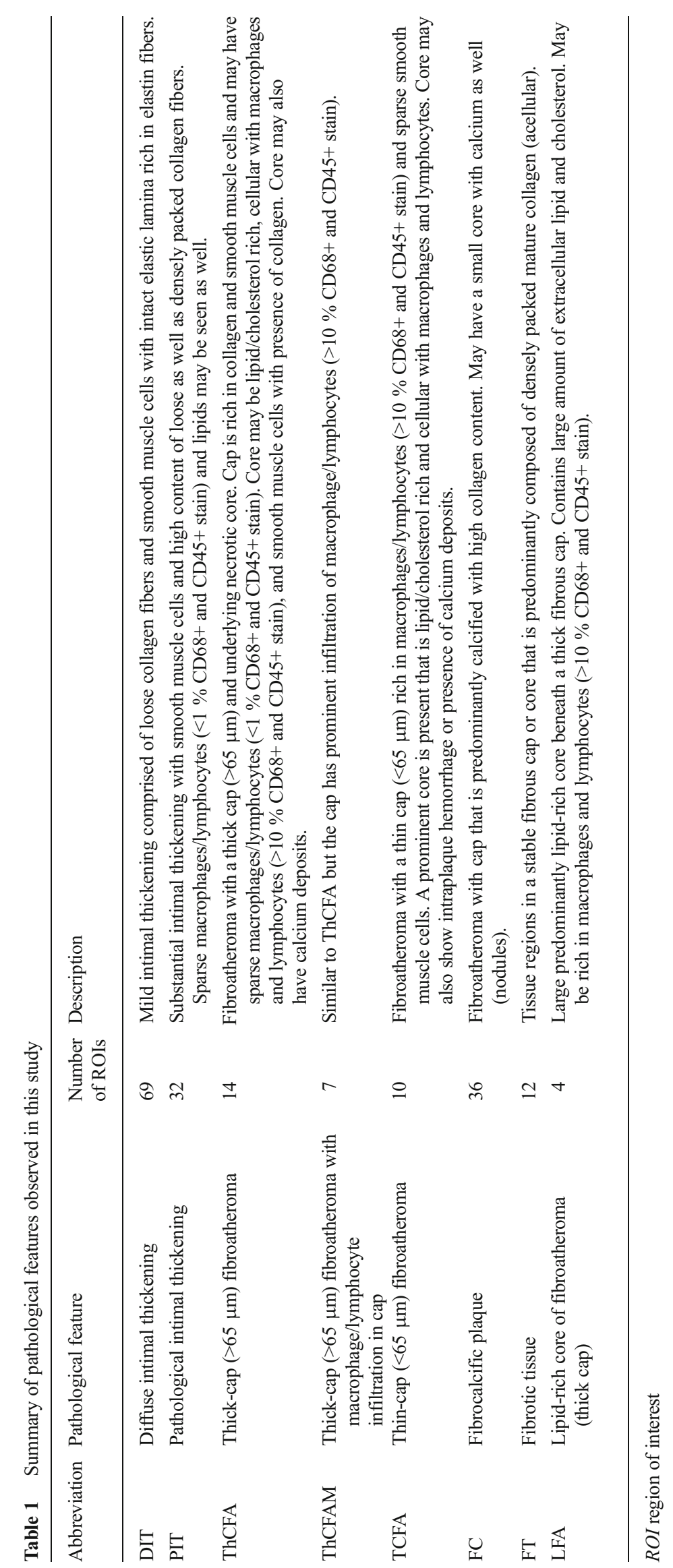


Fig. 1 FLIm-IVUS data visualization. a $\mathrm{CH} 1$ fluorescence lifetime values mapped on to 3-D lumen surface. Three coregistered IVUS frames are shown. b Longitudinal IVUS image with $\mathrm{CH} 1$ fluorescence lifetime values mapped on to the lumen surface. Lower lifetime values indicate the presence of macrophages and lipid in plaque while higher lifetime values relate to increased collagen content. IVUS allows identification of calcium and lipid cores based on high and low echogenicities, respectively
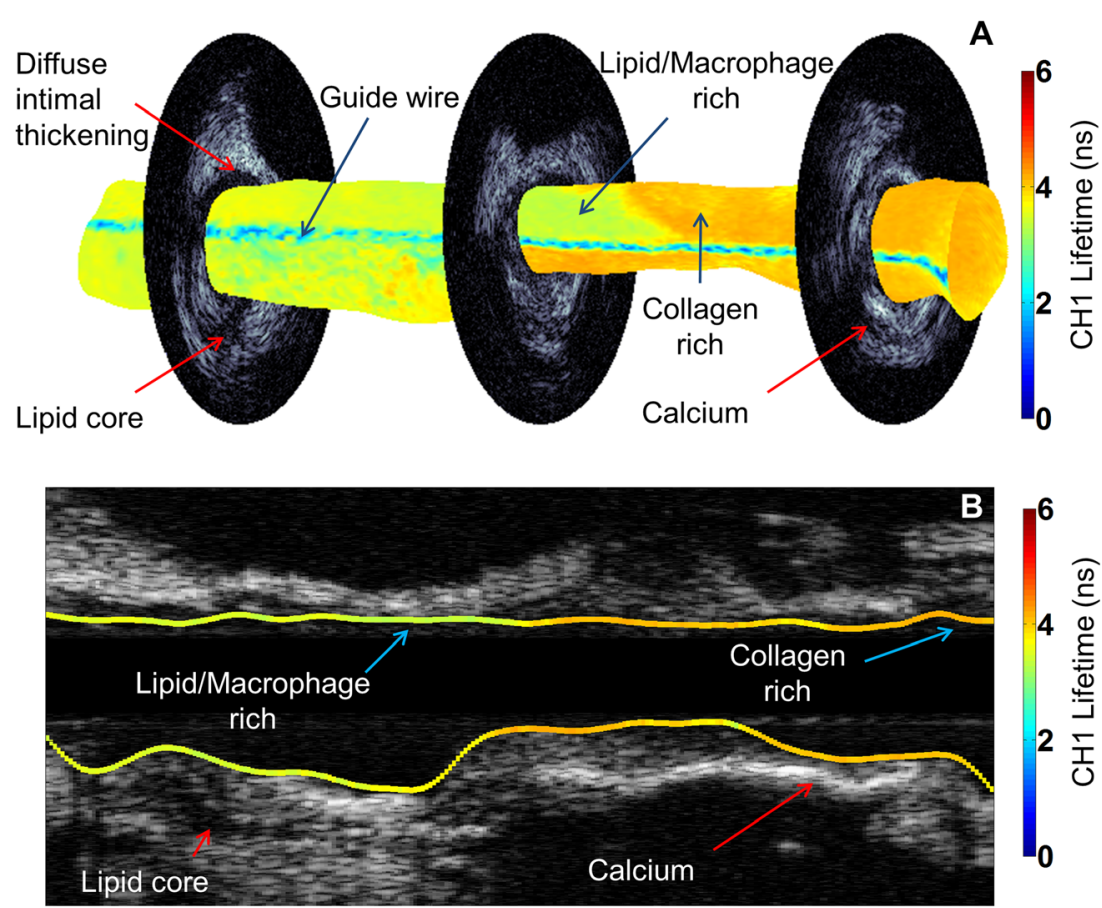

\section{FLIm-IVUS Data and Pathology}

Co-registered FLIm-IVUS images and histopathology data depicting different pathological features are shown in Fig. 2. Figures 3 and 4 summarize distributions of select statistically significant imaging parameters in relation to the pathological features. Data in this section are reported as mean $\pm \mathrm{SD}$ along with confidence intervals at $99 \%$ confidence level. Figure 2a shows a case with diffuse intimal thickening (DIT). Fluorescence lifetime values are seen to be uniform (CH1 lifetime for entire data set for DIT, $3.71 \pm 0.34 \mathrm{~ns}$ (3.71$3.72 \mathrm{~ns})$ ) over the entire lumen surface with IVUS showing narrow to no intimal thickening. Figure $2 b$ shows a case with ThCFA and LFA. A thick fibrous cap is seen in IVUS (small arrow), and lifetime values in this region are higher $(\mathrm{CH} 1$ lifetime for entire data set for ThCFA, $4.27 \pm 0.33$ ns (4.25$4.28 \mathrm{~ns})$ ) due to increase in collagen content as seen in histopathology. LFA (large arrow) is seen on IVUS as hypoechoic region (IB for entire data set for LFA, $-80.2 \pm 3.4 \mathrm{~dB}(-80.3-$ $-80.2 \mathrm{~dB})$ ). Figure $2 \mathrm{c}$ shows a case with pathological intimal thickening (PIT, small arrow) and fibrocalcific plaque (FC, large arrow). PIT is seen with larger intimal thickening in IVUS and has higher average fluorescence lifetime ( $\mathrm{CH} 2$ lifetime for entire data set for PIT, $4.28 \pm 0.19 \mathrm{~ns}(4.28-4.30 \mathrm{~ns})$ ) compared to DIT (Fig. 4). FC is seen as hyperechoic region in IVUS (IB for entire data set for FC, $-57.5 \pm 6.6 \mathrm{~dB}(-57.6-$ $-57.5 \mathrm{~dB})$ ) with loss of signal and shadowing behind the area of interest. The amount of fibrous tissue present in FC can be correlated to an increase in overall lifetime similar to PIT. However, FLIm alone is not able to distinguish between FC and fibrotic tissue (FT) due to similar collagen content, but the two features can be differentiated using IVUS (Fig. 4). Figure $2 \mathrm{~d}$ shows a case with TCFA (both arrows) with intraplaque hemorrhage (large arrow). The spatial resolution of IVUS does not allow visualization of the thin cap. However, there is a significant decrease in fluorescence lifetime values in both regions ( $\mathrm{CH} 1$ lifetime for entire data set for TCFA, $3.44 \pm 0.44 \mathrm{~ns}(3.41-3.47 \mathrm{~ns})$ ) due to low collagen content and high lipid and macrophage content (CD68 stained pathology not shown). Intraplaque hemorrhage is often observed as low-echoic region in IVUS and may be confused with LFA. Figure 2e shows a case with FC which correlates to high backscatter signal in IVUS (arrow). CH1 lifetime values are higher than those observed in DIT (CH1 lifetime for entire data set for FC, $3.98 \pm 0.39$ ns (3.96-3.99 ns)). Figure 2 f shows a case with FT and DIT where the region with FT has higher average lifetime values ( $\mathrm{CH} 1$ lifetime for entire data set for FT, $4.19 \pm 0.29$ ns (4.17-4.20 ns)) than DIT. IVUS can distinguish between DIT and FT based on IT but not backscatter data alone (Fig. 4). Figure $2 \mathrm{~g}$ shows a case with ThCFAM and LFA. Lowering of lifetime values is seen in the region infiltrated by macrophages (small arrow) as confirmed on a CD68stained histopathology section ( $\mathrm{CH} 1$ lifetime for entire data set for ThCFAM, $3.49 \pm 0.71 \mathrm{~ns}(3.44-3.55 \mathrm{~ns})$ ). IVUS is not able to detect the presence of macrophages in fibrous caps but allows visualization of the plaque burden underneath (large arrow). Distributions for select FLIm and IVUS imaging parameters (post hoc Tukey's test $p<0.001$, as shown in Fig. 3) illustrate the ability of FLIm to distinguish between ThCFA and TCFA and detecting the presence of macrophages in fibrous caps (ThCFA vs. ThCFAM). These results (Figs. 3 and 4) emphasize that IVUS and FLIm together allow 

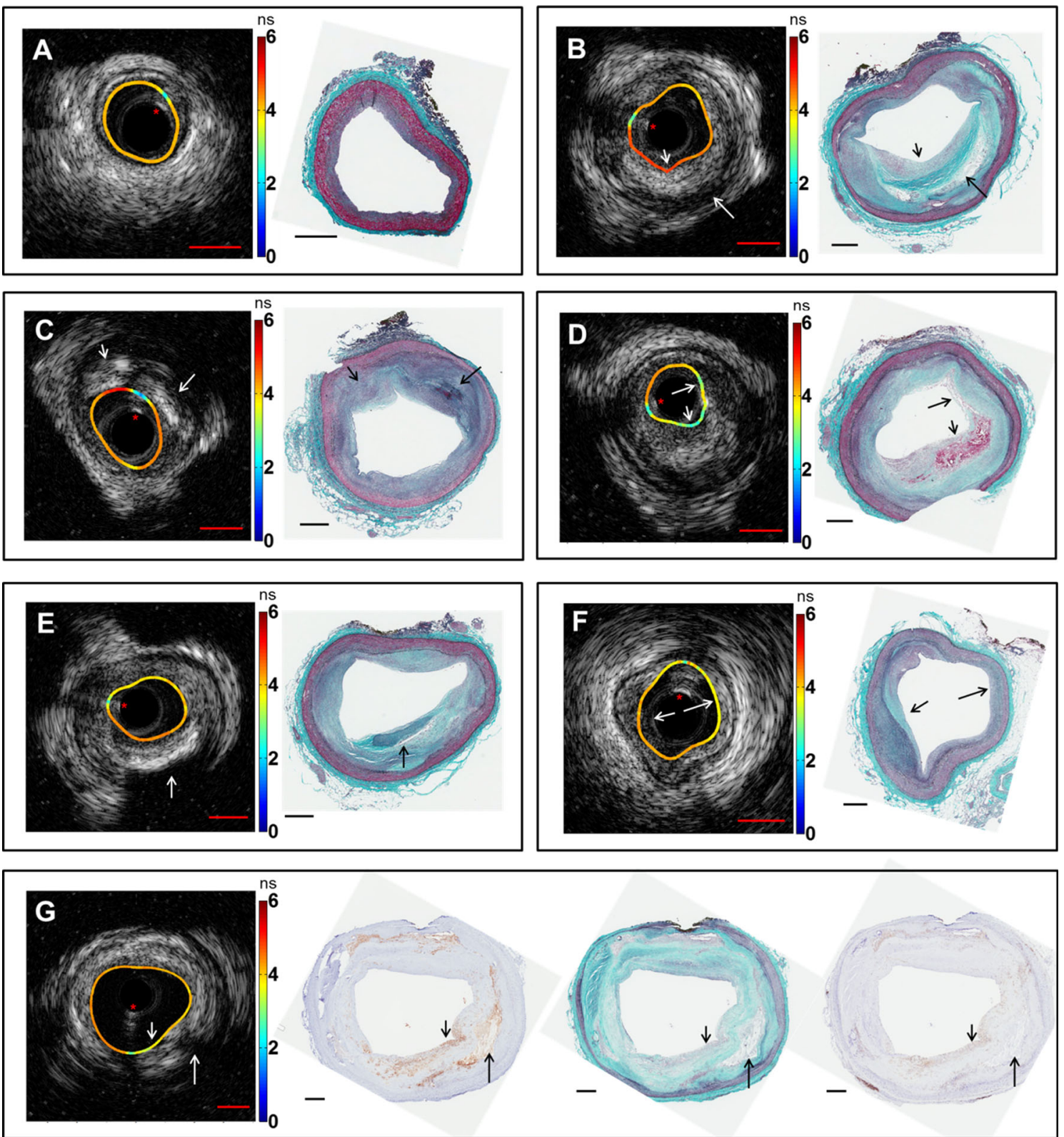

Fig. 2 Representative images of co-registered IVUS, FLIm, and histology data showing different pathological features. Lifetime values (in ns) mapped onto the lumen surface are either from $\mathrm{CH} 1$ or $\mathrm{CH} 2$. Guide wire artifact is highlighted with a red asterisk. a DIT with uniform distribution of lifetime values over the lumen surface. IVUS shows minimal intimal thickening. b ThCFA and LFA. The thick cap (small arrow) is seen on both histology and IVUS with an underlying lipid-rich core (large arrow). Lifetime values at the cap region are higher due to increased amount of fibrous collagen. $\mathbf{c}$ PIT (small arrow) and FC (large arrow) are seen with varying echogenicities on IVUS as well as different lifetime values. d TCFA with intraplaque hemorrhage is seen with distinct lowering in

lifetime values (arrows). IVUS is able to show burden of the TCFA core. e FC (arrow) is seen on IVUS as a region of high backscatter with a shadow. f FT is seen (small arrow) with increased lifetime values due to increase in collagen content as compared to regions with DIT (large arrow). $\mathbf{g}$ ThCFAM is seen with a fibrous cap (small arrow) rich in macrophages as confirmed in CD68-stained section (middle) along with CD45-stained section (right). Lipid-rich core (large arrow) is also seen on both IVUS and histology. Lowering in lifetime values is observed over the fibrous cap rich in macrophages compared to the surrounding regions in the lumen. Scale bars (red) on IVUS are $1 \mathrm{~mm}$, and scale bars on histology (black) are $0.5 \mathrm{~mm}$

distinguishing between fibrolipidic plaques (TCFA, ThCFA, ThCFAM) from stable fibrous plaques (PIT, FT). Distribution of different imaging parameters across all pathological features can be appreciated from Fig. 4. A total of 47 imaging parameters ( 5 from IVUS and 42 from FLIm) were investigated for statistical significance. Of these, 39 were found to be

able to distinguish between at least a pair of pathological features if not more. We show a select set of parameters (Figs. 3 and 4) that show the ability of FLIm and IVUS to distinguish between all the different pathological features. However, all the 39 statistically significant parameters were employed to train an SVM classifier. 
Fig. 3 FLIm and IVUS

parameter distribution for different pathology feature pairs. Parameters shown here are few of many that were found to be statistically different (post hoc Tukey's test $p<0.001$ ) between the pairs of pathological features (described in Table 1)
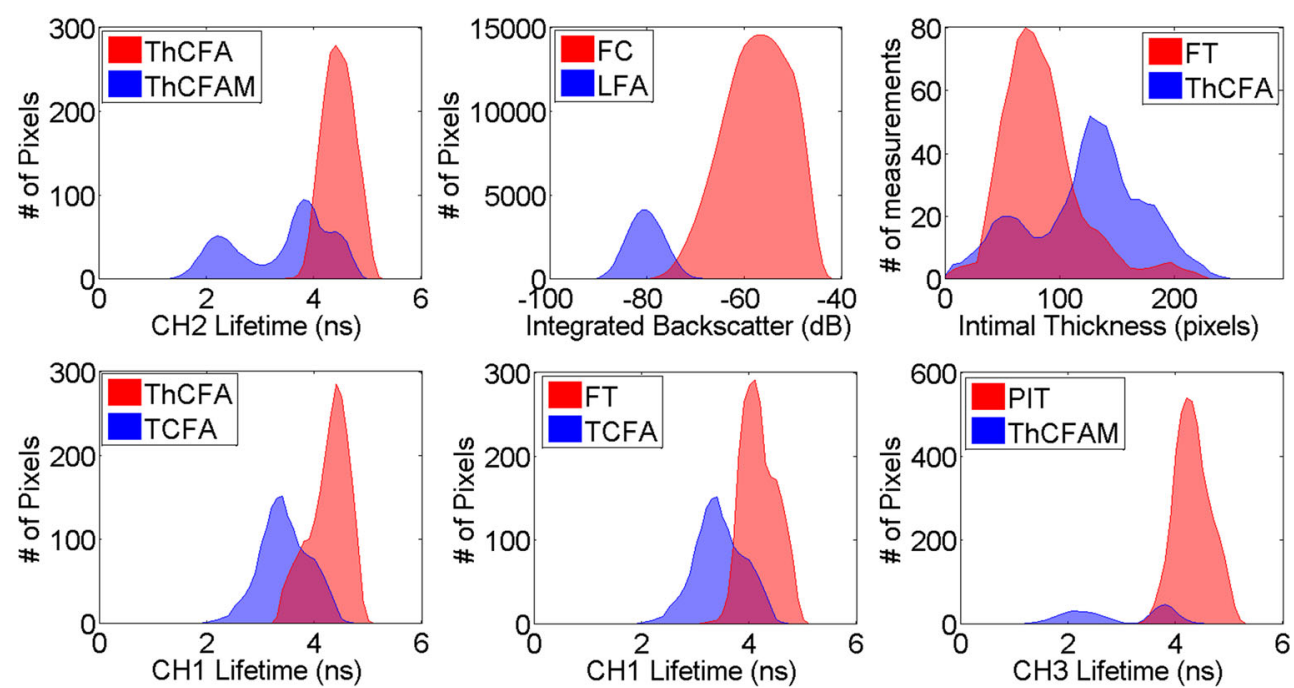
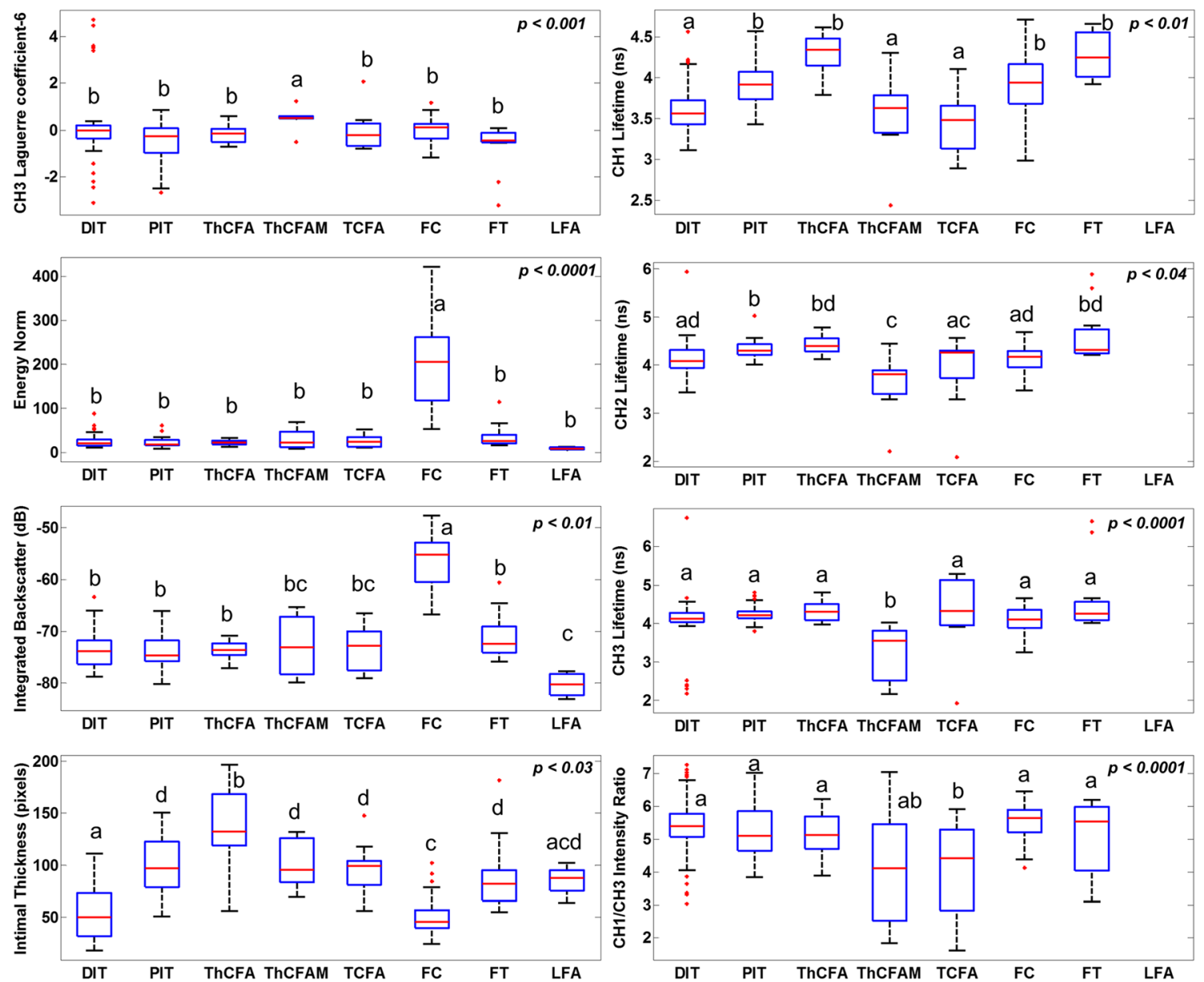

Fig. 4 Box-whisker plots of select FLIm and IVUS parameters across all pathological features (described in Table 1). The red bars represent the median value, the edges of the blue box are the 25th and 75th percentiles, the whiskers extend to 1.5 times the blue box height, and outliers are plotted as red dots. Each box is labeled with one or more letters ( $a, b$, $c$, or $d$ ). Boxes sharing no common letter are statistically different from one another (post hoc Tukey's test $p$-value printed on upper right corner

of each plot). Numerous other parameters were found to be able to discriminate between different pathological features; however, only a few parameters are shown here. No FLIm data are available for LFA due to limited penetration of UV light in tissue $(200-250 \mu \mathrm{m})$. These parameters along with others (not shown) allow discriminating between any given pair of pathological features 


\section{Classification Results}

Classification results are summarized in Table 2. A total of 184 ROIs attributed to different pathological features were used in the analyses. Thirty-nine of the 42 imaging parameters that were statistically significant (mixed effects ANOVA, $p<0.05$, post hoc Tukey's test, $p<0.05$ ) were used to train SVM classifiers from FLIm, IVUS, and FLIm-IVUS data, respectively. Figure 5 shows 3 -D scatter plots showing the data and the SVM classifier hyperplane for two features: ThCFA and DIT. The plots show an SVM built using three parameters; however, the overall classification was performed using SVMs built with all the statistically significant parameters. The plots illustrate the improvement in sensitivity when a classifier is trained using both IVUS and FLIm parameters as opposed to one or the other alone.

IVUS imaging allowed reliable detection of FC and LFA as compared to FLIm alone. FLIm allowed detection of ThCFA, ThCFAM, and TCFA as observed from parameter distributions in Figs. 3 and 4. The sensitivity of detecting ThCFA and ThCFAM improved when both FLIm and IVUS data are used as compared to FLIm alone due to IT information available from IVUS. The classifier trained using FLIm parameters does not include the feature LFA, as no FLIm data are available for this feature due to limited penetration of UV light in tissue. As a result, the FLIm classifier appears to have better performance than the FLIm-IVUS classifier for certain features.

\section{Discussion}

This study demonstrates for the first time that FLIm, a labelfree spectroscopic rotational intravascular imaging technique, can complement morphological information from conventional IVUS with compositional information from the sub-intimal surface. FLIm-IVUS results from human LAD coronary arteries show the ability of this bimodal approach to detect different pathological features (Table 1) that are a subset of the modified classification scheme of Virmani et al. for atherosclerotic lesions [9]. Specifically, results (Figs. 3 and 4) show that FLIm allows differentiating between luminal areas histopathologically classified as TCFA from those classified as ThCFA as well as identifying macrophage infiltration in fibrous caps (ThCFAM). The clinical importance of detecting these plaque sub-types has been well emphasized [16]. Moreover, FLIm-IVUS data can be visualized concurrently (Figs. 1 and 2 and Video1.avi in the supplemental material) to comprehend both luminal composition and morphology of vessels simultaneously for enhanced plaque characterization that may serve as an invaluable tool to study plaque progression.

Compositional changes in tissue can be tracked by quantifying the autofluorescence of tissue components [17]. In this work, FLIm quantifies changes in fluorescence decay dynamics (e.g., average lifetime values and Laguerre coefficients) as a function of changes in arterial tissue composition (e.g., collagen, elastin, and lipids including cholesteryl oleate in macrophages). Thus, the accumulation of macrophages in the fibrotic cap and the co-localization of lipids change the overall fluorescence signature measured from tissue. While these compositional changes can only be assessed within 200 $250 \mu \mathrm{m}$ deep into the plaque due to limited penetration of UV light in tissue [13,14], they provide valuable information about features in the diseased intima that have been associated with plaque instability and rupture. More importantly, for a plaque with a thick cap (ThCFA), FLIm will only be able to

Table 2 Classification results summary

\begin{tabular}{|c|c|c|c|c|c|c|c|c|c|}
\hline \multirow[t]{2}{*}{ Feature } & \multicolumn{3}{|l|}{ IVUS } & \multicolumn{3}{|l|}{ FLIm } & \multicolumn{3}{|c|}{ FLIm-IVUS } \\
\hline & SN (\%) & SP (\%) & PPV (\%) & $\mathrm{SN}(\%)$ & SP (\%) & PPV (\%) & $\mathrm{SN}(\%)$ & SP (\%) & PPV (\%) \\
\hline DIT & 81 & 81 & 71 & 100 & 96 & 93 & 87 & 100 & 100 \\
\hline PIT & 50 & 84 & 40 & 94 & 98 & 91 & 84 & 100 & 100 \\
\hline ThCFA & 29 & 99 & 80 & 57 & 100 & 100 & 86 & 100 & 100 \\
\hline ThCFAM & 0 & 100 & $\mathrm{NaN}$ & 71 & 100 & 100 & 86 & 100 & 100 \\
\hline TCFA & 0 & 100 & $\mathrm{NaN}$ & 90 & 100 & 100 & 80 & 100 & 100 \\
\hline $\mathrm{FC}$ & 100 & 99 & 97 & 44 & 99 & 94 & 100 & 99 & 97 \\
\hline FT & 0 & 100 & $\mathrm{NaN}$ & 100 & 90 & 40 & 92 & 99 & 92 \\
\hline LFA & 100 & 91 & 19 & 0.0 & 100.0 & $\mathrm{NaN}$ & 100 & 92 & 21 \\
\hline Mean & 45 & 94 & 61 & 70 & 98 & 88 & 89 & 99 & 89 \\
\hline
\end{tabular}

DIT diffuse intimal thickening, PIT pathological intimal thickening, ThCFA thick-cap fibroatheroma, ThCFAM thick-cap fibroatheroma with macrophage/lymphocyte infiltration in cap, TCFA thin-cap fibroatheroma, $F C$ fibrocalcific plaque, $F T$ fibrotic tissue, $L F A$ lipid-rich core of fibroatheroma, $S N$ sensitivity, $S P$ specificity, $P P V$ positive predictive value, IVUS intravascular ultrasound, FLIm fluorescence lifetime imaging, NaN not a number (corresponds to division by zero) 


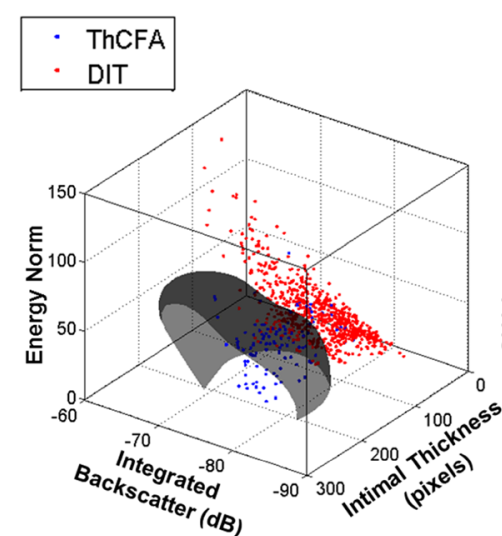

A IVUS $(28.6 \%)$

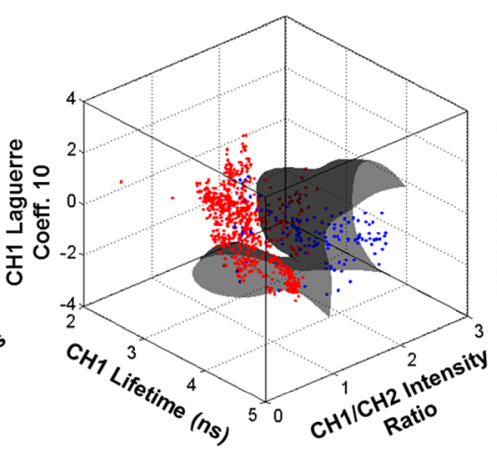

B FLIm (57.1\%)

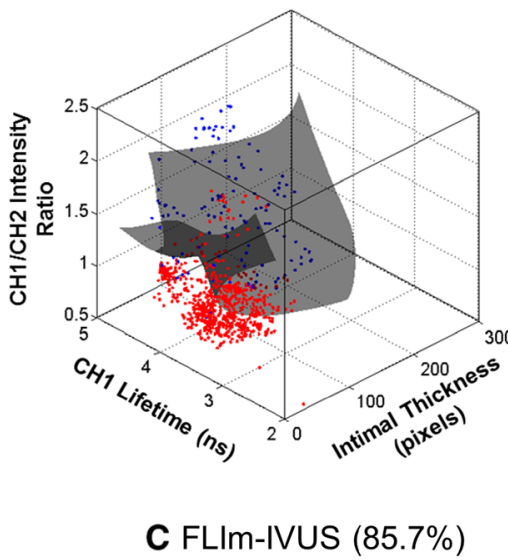

hyperplane (gray) acts as a decision boundary for classification purposes. ThCFA detection sensitivity (reported in parenthesis) improves when both FLIm and IVUS parameters are used, enforcing the benefit of a bimodal imaging technique

\section{Study Limitations}

Current configuration of the intravascular catheter and system requires that the FLIm-IVUS data be gathered sequentially rather than simultaneously. Thus, data acquisition and processing is rather long ( $\sim 6 \mathrm{~min})$. The use of graphics processing units to perform the same task can significantly reduce this time to allow real-time analysis $(<1 \mathrm{~min})$ in our future system. Moreover, our new catheter under development enables simultaneous FLIm-IVUS imaging by integrating the fiber optic and transducer on the same rotational axis. The registration error between FLIm and IVUS in the current study due to sequential imaging was found to be $\sim 18^{\circ}$ from phantom imaging experiments. As a result, ROIs defined by the pathologist that were less than $30^{\circ}$ in span were not used in the study. However, this error will be avoided for future studies using the new catheter design.

Coronary segments in this study were imaged in saline and not in blood. In vivo imaging, however, will be performed by clearing the vessel of blood as is done during OCT imaging. Potential inadequate flushing or presence of blood may affect the FLIm spectroscopic findings which need to be quantified in future in vivo testing of this technique. Current work in progress is investigating flushing techniques to acquire FLIm-IVUS images in vivo in a pig using the new catheter system noted above. Flushing-related complications such as arrhythmia and hypotension that may arise will be addressed by developing a custom IVUS system to increase the pullback speed and reduce flushing times. The limited penetration of UV light in tissue confines the ability of FLIm to interrogate only the biochemical composition of the luminal surface of the arterial wall. However, the compositional information gathered from this region is seen to be beneficial in complementing morphological data from IVUS in this study. 
Relative comparison of performances of various intravascular imaging techniques is shown in Table $\mathrm{T} 1$ in the supplemental material. The spatial resolution of FLIm used in this study is in the order of hundreds of microns $(160 \mu \mathrm{m})$ but proves to be sufficient for imaging luminal surface features.

\section{Conclusions}

This article reports the first results demonstrating the feasibility of a bimodal label-free tissue diagnostic technique combining rotational FLIm and IVUS for intravascular assessment of human coronary plaques. Current results demonstrate that FLIm technique can complement conventional IVUS and enable a better detection of various relevant pathological features that cannot be detected by IVUS alone. FLIm, through changes in tissue lifetime values, is capable of detecting the presence of macrophages in fibrous caps which is a prognostic factor for vulnerable plaques. It can also distinguish between TCFA and ThCFA amongst other pathological features. The changes in lifetime values are attributed to changes in lipid, macrophage, and collagen content in the intima. FLIm results coupled with morphological information from IVUS create a robust tool for staging plaques in arterial vessels. FLIm-IVUS bimodal data can be visualized in a clinical setting in 3-D or as cross-sectional images to aid the physician in the decisionmaking process during PCI. Results from this study lay the foundation for the future clinical applicability of FLIm-IVUS imaging to characterize plaques in vivo in real time, initially in a pig atherosclerotic model and, subsequently, in humans.

Acknowledgments We thank Dr. Abigail Dean and Mr. Kenneth Bedi from the Perelman School of Medicine at the University of Pennsylvania for their assistance in procuring the coronary artery samples, Dr. Diego R. Yankelevich for help with the FLIm optical setup, and Dr. Blythe DurbinJohnson for help with statistical analyses supported by the National Center for Advancing Translational Sciences, National Institutes of Health, through grant \#UL1 TR000002. We acknowledge Boston Scientific Corporation for providing us with the IVUS system used in this study.

Funding The funding of this study is from National Institutes of Health grant R01-HL67377 and the training program in molecular imaging grant 5T32EB003827.

\section{Disclosures None.}

Human Subjects/Informed Consent Statement No human or animal studies were carried out by the authors for this article.

Open Access This article is distributed under the terms of the Creative Commons Attribution 4.0 International License (http:// creativecommons.org/licenses/by/4.0/), which permits unrestricted use, distribution, and reproduction in any medium, provided you give appropriate credit to the original author(s) and the source, provide a link to the Creative Commons license, and indicate if changes were made.

\section{References}

1. Shah, P. K. (2007). Molecular mechanisms of plaque instability. Current Opinion in Lipidology, 18(5), 492-499. doi:10.1097/ MOL.0b013e3282efa326.

2. Puri, R., Worthley, M. I., \& Nicholls, S. J. (2011). Intravascular imaging of vulnerable coronary plaque: current and future concepts. Nature Reviews Cardiology, 8(3), 131-139. doi:10.1038/nrcardio. 2010.210.

3. Schultz, C. J., Serruys, P. W., van der Ent, M., Ligthart, J., Mastik, F., Garg, S., et al. (2010). First-in-man clinical use of combined near-infrared spectroscopy and intravascular ultrasound: a potential key to predict distal embolization and no-reflow? Journal of the American College of Cardiology, 56(4), 314. doi:10.1016/j.jacc. 2009.10.090.

4. Miyamoto, Y., Okura, H., Kume, T., Kawamoto, T., Neishi, Y., Hayashida, A., et al. (2011). Plaque characteristics of thin-cap fibroatheroma evaluated by OCT and IVUS. JACC. Cardiovascular Imaging, 4(6), 638-646. doi:10.1016/j.jcmg. 2011.03.014.

5. Tearney, G. J., Yabushita, H., Houser, S. L., Aretz, H. T., Jang, I. K., Schlendorf, K. H., et al. (2003). Quantification of macrophage content in atherosclerotic plaques by optical coherence tomography. Circulation, 107(1), 113-119. doi:10.1161/01.cir.0000044384. 41037.43.

6. Phipps, J. E., Sun, Y., Fishbein, M. C., \& Marcu, L. (2012). A fluorescence lifetime imaging classification method to investigate the collagen to lipid ratio in fibrous caps of atherosclerotic plaque. Lasers in Surgery and Medicine, 44(7), 564-571. doi:10.1002/1sm. 22059.

7. Phipps, J., Sun, Y., Saroufeem, R., Hatami, N., Fishbein, M. C., \& Marcu, L. (2011). Fluorescence lifetime imaging for the characterization of the biochemical composition of atherosclerotic plaques. Journal of Biomedical Optics, 16(9), 096018. doi:10.1117/1. 3626865.

8. Ma, D., Bec, J., Yankelevich, D. R., Gorpas, D., Fatakdawala, H., \& Marcu, L. (2014). Rotational multispectral fluorescence lifetime imaging and intravascular ultrasound: bimodal system for intravascular applications. Journal of Biomedical Optics, 19(6), 066004. doi:10.1117/1.JBO.19.6.066004.

9. Virmani, R., Kolodgie, F. D., Burke, A. P., Farb, A., \& Schwartz, S. M. (2000). Lessons from sudden coronary death: a comprehensive morphological classification scheme for atherosclerotic lesions. Arteriosclerosis, Thrombosis, and Vascular Biology, 20(5), 12621275.

10. Gorpas, D., Fatakdawala, H., Bec, J., Ma, D., Yankelevich, D. R., Qi, J., et al. (2015). Fluorescence lifetime imaging and intravascular ultrasound: co-registration study using ex vivo human coronaries. IEEE Transactions on Medical Imaging, 34(1), 156-166. doi:10. 1109/TMI.2014.2350491.

11. Katouzian, A., Sathyanarayana, S., Baseri, B., Konofagou, E. E., \& Carlier, S. G. (2008). Challenges in atherosclerotic plaque characterization with intravascular ultrasound (IVUS): from data collection to classification. IEEE Transactions on Information Technology in Biomedicine, 12(3), 315-327.

12. Liu, J., Sun, Y., Qi, J., \& Marcu, L. (2012). A novel method for fast and robust estimation of fluorescence decay dynamics using constrained least-squares deconvolution with Laguerre expansion. Physics in Medicine and Biology, 57(4), 843-865. doi:10.1088/ 0031-9155/57/4/843.

13. Richards-Kortum, R., \& Sevick-Muraca, E. (1996). Quantitative optical spectroscopy for tissue diagnosis. Annual Review of Physical Chemistry, 47(1), 555-606. doi:10.1146/annurev. physchem.47.1.555. 
14. Wilson, B. C., \& Jacques, S. L. (1990). Optical reflectance and transmittance of tissues - principles and applications. IEEE Journal of Quantum Electronics, 26(12), 2186-2199. doi:10.1109/3.64355.

15. Lindstrom, M. J., \& Bates, D. M. (1988). Newton-Raphson and Em algorithms for linear mixed-effects models for repeated-measures data. Journal of the American Statistical Association, 83(404), 1014-1022.
16. Libby, P., Ridker, P. M., \& Maseri, A. (2002). Inflammation and atherosclerosis. Circulation, 105(9), 1135-1143. doi:10.1161/ hc0902.104353.

17. Berezin, M. Y., \& Achilefu, S. (2010). Fluorescence lifetime measurements and biological imaging. Chemical Reviews, 110(5), 2641-2684. doi:10.1021/cr900343z. 